\title{
Improvement of Semantic Search Results with Providing an Updatable Dynamic User Model
}

\author{
Samira Karimi-Mansoub \\ Department of Computer Engineering \\ Hacettepe University, Turkey
}

\author{
Rahem Abri (BiDEB) \\ Department of Computer Engineering \\ Hacettepe University, Turkey
}

\begin{abstract}
The current generation of search engines is severely limited in its understanding of the user's intent and the Web's content and consequently in matching the needs for information with the vast supply of resources on the web [10]. The search engines are evolving from the keyword matching search in general to conception match search personalized by user. In this article, is tried to use the semantic search engines to improve the efficiency level of personalization process. Since the general problem in the search results produced by semantic search is the overload information and the mismatch between the results and corresponding requirements needed by the user, so there is an attempt to troubleshoot the problems by applying user model to some extent. The research project at hand is oriented to collect personalized data to be displayed and create the user model. Models create a structure to display the information on the basis of the user's priorities. The initial step to develop a user modeling is to collect and compile user's interests. In this paper, is studied how to infer a user's interest from the user's search context and use the inferred implicit user model for personalized search.
\end{abstract}

The main focus in this research, is on the embetterment of the efficiency of the results produced by the semantic search of updated user model. Since the low efficiency of personalized processes by the user model is due to low level factors and components by which user model is built up and the algorithm by which the model is updated, so in this research is tried to improve the efficiency of user models by investigating and modifying these cases and as a result, the improvement of search results. This paper presents to research fields of user model and semantic search and also it attempts to show how recent semantic research procedures in web development can intermingle with modern technologies of user model. Some experiments were carried out to evaluate the suggested procedures for user profile and the results showed some improvement in the user's satisfaction when the user used their profiles to personalize the results from semantic search.

\section{Keywords}

Semantic search; implicit user model; Ontology; personalized search; user search history.

Supported by Turkey BIDEB program.

\section{INTRODUCTION}

With advances in communication technology, the amount of electronic information to which the users can have an access increase dramatically. The human needs are not satisfied comprehensively when one uses current technologies in the web. In current web, data are readily stored and published But this type of storing brings about a lot of problems to retrieve and use the data later on. Semantic web is an alternative solution for these problems and its objectives are to share the data so intelligently that they are fathomable not only for human beings but also for machines and devices.

Search engines are tools that are currently used for web search. The current generation of search engines is strictly limited in understanding the user's intentions and web content and also in conformation of user's data requirements to the vast resources of web. The search engines are evolving from the keyword matching search in general to conception match search personalized by user [10].

The unwanted results presented by current search engines are due to the following: 1 . the procedures to search the keywords, heedless of the user's priorities and interests. 2. the semantic gap between the terminology used by the users and recognized by the search engines. This is indicative of the shortcomings in searching and it has to be investigated as a technology with different aspects and factors. The search engines that seek to improve their search results using semantic web are called semantic search engines.

Enhancement in and sporadic evaluation of semantic search tools suggests a promising perspective of promotion in functionality of traditional information retrieval systems. There is a general drawback in retrieving the information in semantic search, i.e., the search results of semantic searchinformation overload and minimal correspondence between results and users' needs. So semantic search by itself cannot meet the users' requirements and there remains the necessity for users to personalize them according to their interests. The personalized search methods using user model can somehow remove the problem.

Although users of this electronic information have access to a rich body of information, only a small fraction of the information is actually relevant to any one user's interest. Thus, there is the problem of determining the part of the information that is of interest to the user while minimizing the amount of search through irrelevant information. Clearly, it can conclude that there is a need for automatic tools that capture information for filtering and control and adjust based on user interest are published [30].

One solution is to develop filters that selectively weed out the irrelevant information based on user preferences. While automatic filtering of information sounds like a wonderful vision, there are many difficulties in determining what information a person would actually want to see [30]. Therefore, the question of information personalization which has recently been under extensive study is raised. One of the results of personalized systems is user model. User modeling can be described as the process of building the personal preferences of the users in terms of user's knowledge about the world, her behavioral aspects, goals, likes and dislike [8]. Models develop a structure to display the information based on the user's priorities. 
The model of the user is generally represented in the form of user profile which captures the personal preferences in a machine proccessable format [8]. There are problems regarding the development of a good model out of user's interests. The most important problem posed here is that what information is available to the system that can infere the user's information requirements?

Many procedures with different levels of efficiency and application have been introduced to elicit the user's interests. But most of the existing systems rely on the user's search history and his/her query to model the user's requirements. According to what was already said, the main challenge to develop a personalized information service is the way the user's interests are inferred. In this research, a dynamic user modeling is created to do a personalized search based on user search history by using a new updatable pattern. This method differ from other existing methods in a way that it develops a pattern for user model that leads to the enhancement in effectiveness and updatablity.

In this research, is concentrated on enhancing the efficiency of user modeling algorithm since the low efficiency of personalized processes by the user model is due to low level factors and components by which user modeling is constructed and the algorithm by which the model is updated and therfore is managed to improve the efficiency of user model and the results of semantic search. In this paper, search results are enriched by the feedback from the user's search behaviors. The information implicitly elicited from the user cannot be directly used to describe the user's interests, but they are methodically interrelated.

The remaining parts are organised as thus: In part 2, extensively is explained the subject matter in greater details. Part 3, review the literature and research in this regard. In part 4 , is stated major algorithm and is suggested a model research method and evaluate the suggested model and in the last part, there is a conclusion and future works and sum up all stated beforehand.

\section{STATING THE PROBLEM}

As mentioned in the introduction, solutions to current problems that exist in the web, is use of user model for personalization process of search results. User model is an integral part of any personalized information retrieval system. The user model should be adaptable in order to capture the change in information needs of the users [8].

Two main problems are presented here:

1. The presentation of user model that not only deals with the user's needs but also has high effectiveness.

2. Updatablity of this model according to the needs and priorities of the user.

Owing to the fact that in the investigation of user model and its development, different factors like the ways to explicitly or implicitly collect user's interests, to consider short- and longterm interests and so forth ought to be borne in mind, the factors leading to higher effectiveness are highly important. This research has the potential merit to suggest how to periodically update the user model in addition to developing a new pattern in user model. Using this model, one can improve the accuracy of search and effectively support personalized services.
In this research, user model is used to improve the results of semantic search. First, a semantic search is done on the user's query, then the results are filtered through user's intertests and priorities. Finally, user model is updated. The final yield is the search result that conforms to what was supposed to be looked up. There is an endeavor to use semantic search engines instead of regular search engines in this research. Semantic search engines search the information according to semantic relations based on semantic web; thence, an increase in efficiency.

In this paper is developed the user model by examining different factors and research literature, analysing what contexts to be read and what content to be contained. To foresee which pages are to be read on the basis of the previously read pages, is used a measurement scale of parallelism between new pages and old pages and the resemblance between the pages provided us with the criterion to estimate the user's interests. In this research, is suggested a technique to obtain and update user model by analysing the user's information from user's search history. Some experiments were carried out to evaluate suggested technique and the results were indicative of user's satisfaction when they used their profiles for personalization.

\section{RELATED WORKS}

In this research, is used one application of semantic web which is called semantic search and is tried to optimise the search process in the web using user model. In [16] is presented semantic search which is built on these supporting technologies and is designed to improve traditional web searching. In this paper, provide an overview of TAP, the application framework upon which the Semantic Search is built. In semantic search are used of different methods. Ontologies are most methods used in the semantic search [2]. Since semantic search based on ontology also cannot meet the user's requirements by itself, the necessity to personalize the information ought to be conformed to user's interests. Web personalization is process selection, preparation and delivery of Web concepts for a given user is considering his specific needs and priorities. The goal of personalization in the paper [3] is to build user model and its use in semantic search.

Personalization of web sites has become an important issue in web modeling methods [1][12][13][4][5][6], due also to the effect of the diversity of personalization policies over all the development cycle of applications. Personalization is used in various fields that mention some of them. Many of these methods tackle very similar problems and use conceptually similar techniques. Despite of this, even with the same (personalization) requirements the final implementations differ [38]. For example, the paper [4] expressed the need to the use of personal services in application areas like ecommerce and $\mathrm{m}$-commerce that this area requires ubiquitous access to web applications.

In the paper [17] used a different field of personalization. In this paper, is addressed the problem of personalization in question answering (QA) and explain how the user's characteristics can be represented in a QA system via a user model.

One of the most use of personalization, is to build user model for optimize the search process that will also discuss in this study. Currently some search engines provide rudimentary personalization, such as Google Personalized web search [19], which allows users to explicitly describe their interests by selecting from predefined topics, so that those results that match their interests are brought to the top, and My Yahoo! 
search [18], which gives users the option to save web sites they like and block those they dislike [32].

Ontology has been a basis for the construction of a user model in several personalized systems ranging from information delivery systems to Intelligent Tutoring Systems [7][9]. In this section, is provided a brief discussion of a number of such systems. In [26], the user profile is represented as hierarchy of concepts. The concepts are adopted from reference ontology of 4,400 concepts taking the top level categories from Magellan web site.

My planet [11] is ontology based personalized news delivery system. Simple relationships among the concepts inside the domain have been used to filter out information relevant to the user. In [31] ontology is used to improve the performance of personalized information retrieval. This context, which includes the interest rate and the associated user is automatically updated.

The research works differ in the way they represent the user profile and the adaptively of the system. But even among the adaptive systems, the algorithm for learning users interest varies [8].In [32] use of implicit user modeling for personalized search. In this paper, has presented a client-side web search agent called UCAIR that perform necessary operations for implicit feedback. UCAIR is a web browser plug-in that acts as a proxy for web search engines. Currently, it is only implemented for Internet Explorer and Google, but it is a matter of engineering to make it run on other web browsers and interact with other search engines.

There are two remarkable advantages on this. First, the user does not need to worry about the privacy infringement, which is a big concern for personalized search [22]. Second, both the computation of personalization and the storage of the user profile are done at the client side so that the server load is reduced dramatically [20]. This way, the captured user information always resides on the computer that the user is using, thus the user does not need to release any information to the outside. Client-side personalization also allows the system to easily observe a lot of user information that may not be easily available to a server. Furthermore, performing personalized search on the client-side is more scalable than on the server side, since the overhead of computation and storage is distributed among clients [32]

In [8], is presented an ontology based user modeling strategy in the context of personalized information access. In this paper is adopted a hybrid approach by capitalizing on the features of static and dynamic user profiling strategies. Static user profile specifies the user's interest in a much focused manner and dynamic user profiling adds the feature of adaptability into it. The dynamic user profiling strategy make use of the data sources like usage log and mouse operations that are performed by the users during the browsing sessions. In this paper, is described the representation of the domain knowledge in ontological structure and the use of the domain knowledge in user modeling.

[14] Proposed a system for constructing user models automatically by monitoring the users browsing behaviors in each session. The system keeps track of the usage logs by means of Semantic Web Usage Log Preparation Model (SWULPM). The user model consists of personal ontology which is represented through concept graph [8].

In [21], is studied how a search engine can learn a user's preference automatically based on her past click history and how it can use the user preference to personalize search results. In this paper, ranking mechanism is largely based on a recent work by Haveliwala on Topic-Sensitive PageRank [29]. In this work, instead of computing a single global PageRank value for every page, the search engine computes multiple Topic-Sensitive PageRank values, one for each topic listed in the Open Directory [21].Due to the complexity of the analysis, rating calculations and relationship with the preference vector, this method is difficult to scale to general search engines. There is not also an effective mechanism to combine both the content and the web link structure for personalized search.

Raghavan and Sever [28] use a database of past queries that is matched with the current user query. If a significant similarity with a past query is found, the past results associated with the query are proposed to the user. The research focuses on the similarity measure used to calculate the query-to-query similarity. This cannot be based on traditional word-to-word IR matching functions, such as the cosine measure, because the short nature of queries makes them particularly susceptible to the vocabulary problems of polysemy and synonymy.

[26] Propose a novel model of user profile that user profile is organized as a hierarchical structure, rather than a set of independent domains. Although the representation of this user profile is innovative, but work does not make use of the characteristics of hierarchical structure (e.g. to split or to merge nodes in the user profile) to capture the dynamics of changes in user's interests. Pretschner's article [26] describes visiting duration of a page is a good candidate to measure the preference.

In [23], [24], [25], and [27] utilize relevance feedback to update the user profile. Although relevance feedback is effective, users are overloaded. In discussion of the changes of user interests, [25] and [27] found that there are two types of the user interests. One is the short-term interest and the other is the long-term interest. The short-term interest is usually caused by a hot news event and vanishes quickly. Relatively, the long-term interest often reflects a real user interest.

In [33], is presented PVA, an adaptive personal view information agent system to track, learn and manage user interests in the Internet documents. PVA consists of three parts: proxy, personal view constructor and personal view maintainer. Proxy logs user's activities and extracts user interests without user intervention. Personal view constructor mines user interests and maps them to a class hierarchy (i.e. personal view). Personal view maintainer synchronizes user interests and personal view periodically. When user interests change, PVA, not only the contents but also the structure of user profile, is modified to adapt the changes. To build a personal view, user interests have to be tracked and categorized and adopted the classification method based on a pre-defined category hierarchy, called world view, as the superset of the category hierarchy of personal view. PVA consults a world view as the reference and extracts the interesting categories to build its personal view. In this study, ACIRD [34][37], is selected as the world view. One of the drawbacks of this article is the failure to consider threshold for openness page.

\section{Presenting Model}

In this part, is presented an algorithm of the suggested model and the process of constructing the user model. The purpose of presenting this model is to enhance the efficiency of the search results by semantic search of updated user model. When a new information is searched, first a semantic search is 
done. Then the results are analysed and weighed against the user model through a process about which will explain in the folowing. If the obtained information through search corresponds with the user's interests, then they return as search results. If the correspondence lacks, these information are replaced with those of previously searched, using a new pattern. This is what is called updating the user modeling. In this paper is tried in this research to use semantic search engines instead of common search engines to have more accurate search results, observing the semantic relations based on semantic web; thence, an increase in efficiency. Due to the dearth of time and higher levels of activities in constructing a user model, in this paper will use present semantic search engine.

In the simplest case, a user modeling includes registration form or a questionaire with explicit statement of user's interests by the user himself/herself. In more sophisticated and developed cases, a user model includes a dynamic information structure that obtains and estimates such profile (i.e., background) information about the user as his/her knowledge level, the rate of resemblance between his/ her field of interest and the user's behaviors over a period of time. Although this technique is immensely accurate, users usually waste a lot of their time specifying their needs or modifying them explicitly. They are not often able to utilize these techniques effectively or they find them confusing and unreliable. Apart from the need for extra time to be spent on these processes, the users have to shoulder more responsibilities. So the efficiency of explicit techniques may become somehow limited. So there is a need for another technique to personalize search results that develop algorithms which infer these needs in an implied way instead of specifying the user's need by explicit queries or manual updating by user's feedback.

Basically, implied feedback techniques design the user's needed information by tracing and displaying the user's behaviors through an explicit process. personalized systems can collect the data used by such server partners as server access logs or search queries or histories or they can gather up the data used by such customer partners as cookies, mouse and keyboard trackers. But implicit techniques have lower accuracy and efficiency. There are techniques that eclectically combine both explicit and implicit ways. In this research, is used implicit techniques to gather up some information about the user by selecting and combining some factors and is tried to enhance the efficiency.

In this paper is used a registration process to allow the user $\log$ in the system before she/ he wants to search anything. This process requires the user to type in username and password to build up a profile for him/ her irrespective of his/ her interests. Thus it doesn't put any burden on the user. Having registered and logged in the system, the user can search in semantic search engine. The first time the user does the searching by queries having some phrases with keywords, the results reflected are the same as the ones presented by semantic search engine. Since no profile or user model has been constructed for the user as yet. But after first search, a profile will be constructed for him/ her and it will be tagged and attached to his/ her username, based on the process about which will explain extensively and from then on, when the user searches, the search results will be filtered through his/ her user model constructed for him/ her.

Obtaining the knowledge and priorities by the user is problematic so as to provide him/ her with personalized aids. The user model presented in this paper is constructed by combining two different factors, i.e., the time during which a user keeps a page open and the content of the pages. In "Figure 1" an overview of this system is presented.

\subsection{User Modeling Design}

The user model presented in part 4 is designed via following steps:

\section{Constructing, Comparison, Updating and Representation}

Constructing: This is the preliminary step to build a profile. In this step, the user's interests are derived through a process from the content of the pages visited by the user.

Comparison: in this step, new results are compared with the user's profile.

Updating: in this step updating and maintaining the user profile is carried out.

Representation: this step intends to display output results.

\subsection{Constructing User Profile}

In this part, for development of a profile from user interested documents, the keywords are extracted from the documents. In this paper, user profile is a category hierarchy where each category represents the knowledge of a domain of user interests. Each category is described through a keyword vector, Creation date and Number of referrals and then is adopted the classification method based on a pre-defined category hierarchy, called world view, as the superset of the category hierarchy of profile. In this paper, is used from world view available in [33]. Access time is an important factor which is studied as a primitive filter.

The time during which a user keeps a page open determines how much the user is interested in that page. Users dedicate more time to the pages they like than to the ones they do not. Of course, one should be attentive to the fact that a user may keep an unwanted page open while he is doing something else. Therefore, is considered two value thresholds - low and high. These thresholds should be logical and help to observe the time factor logically.

A. In this part, first will improvise a time counter for the page which the user opens to calculate the time. In the experiments done in paper 26, a group of 16 users was studied for 26 days. These results showed that $20 \%$ of the visited pages were kept open for less than 5 seconds. And the average search time for one page was 54.49 seconds. And in paper [33], is observed that the pages being visited over the predetermined threshold ( 2 minutes) are sent to be produced and updated. In this part also is combined two thresholds and is been chosen a time threshold between 2 and 15 minutes. 


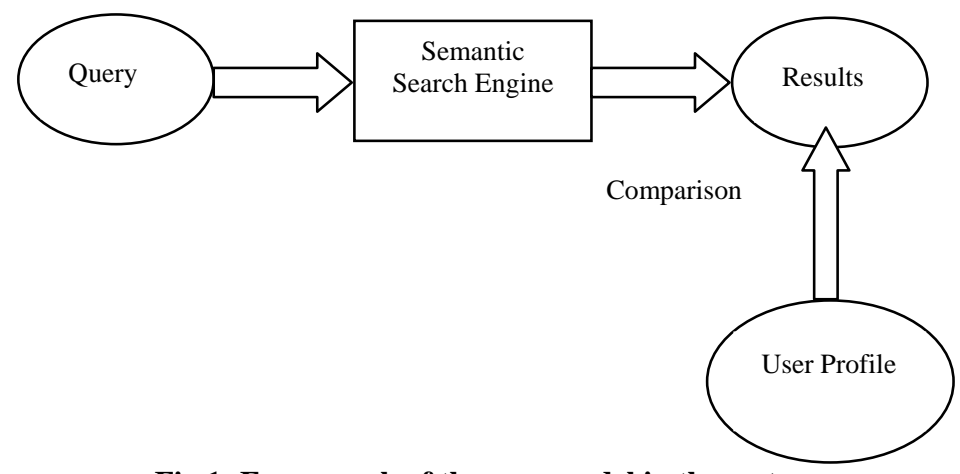

Fig 1: Framework of the user model in the system

After the user closes the page, the time of the page would be equal to the threshold time if the page time $\left(t_{i}\right)$ transgresses the threshold. If the page time $\left(\mathrm{p}_{\mathrm{i}}\right)$ is somewhere between high and low threshold, it is sent to the next stage, i.e., estimation and extraction of keywords.

\section{If $120<t_{i}<900$ [second] then STAGE B.}

B. In this stage, time factor is exerted on the visited pages in a way that one can say one particular page is appropriate and its keywords have to be extracted. A keyword vector has to be calculated for each page. Extracting keyword phrases plays an important role in displaying the user's profile. The trend of keyword phrase extraction and calculation of their weight in a page is calculated according to formula TF-IDF:

$W=T F\left(k_{i}, p\right) \log \frac{n}{D F\left(k_{i}\right)}$

Where $\mathrm{K}_{\mathrm{i}}$ stands for a word in a page

$\mathrm{TF}\left(\mathrm{K}_{\mathrm{i}}, \mathrm{d}\right)$ stands for the frequency of $\mathrm{K}_{\mathrm{i}}$ in a page,

$\mathrm{n}$ is the number of all pages and

$\mathrm{DF}\left(\mathrm{K}_{\mathrm{i}}\right)$ shows the pages that contain $\mathrm{K}_{\mathrm{i}}$

C. Hereby the weight of keyword in user-interested pages are calculated. Now some of the keyword have to be omitted due to their multiplicity in a page. The method is as following: Firstly, the stop word in the keyword list should be crossed out. Then the keywords are sorted out by their weights descending. At last, the highest $\mathrm{N}$ for the word with biggest weight in one particular page is saved in the format of following weights in Matrix M. (According to the formula 2)

$W=\left\{w_{1}, w_{2} \ldots w_{N}\right\}$

$M=\left[\begin{array}{cc}P_{i}, \text { Date } & \left\{\left(w_{1}, k_{1}\right),\left(w_{2}, k_{2}\right), \ldots,\left(w_{N}, k_{N}\right)\right\} \\ \vdots & \vdots\end{array}\right]$

Thus for each visited page, $\mathrm{N}$ keywords with the highest weight according to formula TF-IDF as well as the visiting date are calculated and saved in matrix.

D. Then the parsed keyword vector is sent to an automatic classifier to find the categories that the keyword belongs to. Each obtained category represents a classification path

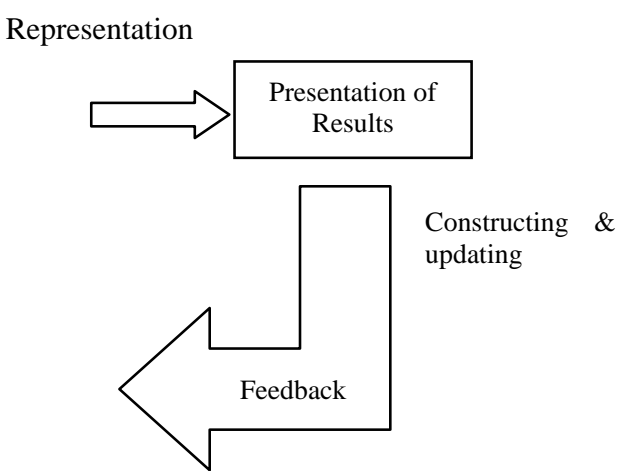

That is a path from the root to the assigned category in the world view. To insert the interesting keywords in a profile,

First is checked that the category exists in the profile. If the category exists, the keyword is inserted in the category directly. If the category does not exist, insert the keyword in the non-root closest ancestor in the profile. If no non-root ancestor exists, the top category of the classification path is created in the profile and subsequently the keyword is inserted in that category and the keywords vector, Creation date and Number of referrals of category is updated. With each visit, number of referrals increases.

As said beforehand, when the user first uses the semantic search engine to search semantically, just the results from the search engine is returned to him/ her. At the very time these results are displayed, user profile is constructed so that they could be used later.

Next time the user enters the website to surf, he/ she is first asked for his/ her username so as to activate the profile peculiar to him/ her. In this step, the user inserts query qh. System returns a series of results, but all these results have to be filtered through user model and thenceforth displayed to the user and at the same time user model be updated based on new results. The comparison between the visited pages and user's profile is explained as following.

\subsection{The Comparison between the Newly Visited Pages and User Profile}

In this step, each page that semantic search engine returns is compared with the previously constructed user model before anything is displayed to the user. That is, the keywords in each page are extracted by the above-mentioned process and compared with the user's profile. If the resemblance rate of keywords of this page with the keywords in user's profile is higher than the threshold, it can be inferred that the user is interested in this particular page because the intended keywords exist in user's profile. Then that page would be returned to the user. This means that a measurement function 
of resemblance rate between the user profile and new page is applied. Finally the results will be displayed in presentation function and for updating the user profile; one has to see to how the user treats these results.

\subsection{Updating and Maintaining User Profile}

The algorithm which is used for updating is an optimal one that takes the two factors of visiting frequency and the time spent into consideration. This algorithm tries to consider the user's long-term and short-term interests and to enhance the efficiency of presented user modeling and also to improve the results of semantic search by omitting the less often used or visited pages. The process of updating the user's profile starts when the search results conform to his/ her profile. In this process, one has to see to how the user treats these results.

To update a user's profile, when a user sees a page and stays there for a longer time than the threshold time, the keyword of that page are extracted and their weight is calculated and are sent to user's profile for updating. In this step, the vector of the keywords in the page is analyzed and all the words are individually compared with user's profile. If they exist in the profile, the numbers of visits go up and the time spent is updated. If they do not exist in the profile, it goes to the appropriate class by its comparison with world view structure and the keyword vector of that class is updated accordingly. And the oldest and least often visited keyword is crossed out of user's profile.

\section{EVALUATION}

In designing and developing an evaluative procedure for personalization, using user model in this paper, there is an intend to evaluate whether filtering the personalized results based on the user's interests and user model presented in this research yields the desired results and if the effectiveness will enhance in comparison to the output from common search engines like Hakia. Of course, evaluating such systems is awfully difficult due to extensiveness of the project and user's wavering behaviors with the systems.

This part is classified in some subparts. First subpart is about criteria, measurement formula and evaluation technique. Next subpart deals with designing the experiments. And finally, the third subpart contains the results of evaluations and experiments along with diagrams and tables.

\subsection{Criteria and the Evaluation Procedures}

In this part, is adopted the following formula for evaluation from paper [33] and by combining formula (3) with a questionnaire in the next part, to evaluate suggested model.

\section{Precision $\frac{\perp D_{\text {filtered } \text {-interesting }} \mid}{\left|D_{\text {filtered }}\right|}$}

Where $D_{\text {filtered }}$ is a set of documents that pass through the filtering, $D_{\text {filtered_interesting }}$ is a subset of $D_{\text {filtered }}$ that is interesting to the user, $\mathrm{D}_{\text {interesting }}$ is a subset of testing documents of a day that are interesting to the user.

\subsection{The Design and Development of the Experiments}

This part start to evaluate and compare the model with that of HAKIA search engine on which the search was in order to evaluate and investigate the efficiency of suggested model using the formulas presented previously.

To experiment the system on this model, it forms a group of 20 subjects. In the first step, each one is asked to separately search a query in hakia search engine and in suggested model of this research. Then the subjects are required to fill in the questionnaire with the help of the first 5, 10, 15 and 20 results provided by search engines. In the second step, the subjects are required to change their queries and do the queries with 1 , $2,4 \ldots 10$. In the next step, is compared the questionnaire results using diagrams and tables. The questions to be asked from the users to construct the profile are as following:

Answer these questions by investigating both results and queries.

I. How many of the hakia search results exist in suggested model search results? $\mathrm{D}_{\text {filtered }}$

II. How many of the search results of suggested model interest you? $\mathrm{D}_{\text {filtered-interesting }}$

These questions are asked for each query separately and the results are gathered up.

\subsection{The Evaluation Results and the Experiments}

Evaluation of first 5, 10, 15 and 20 results of search engines and also for $1,2 \ldots 10$ query, were separately done with the aid of formulas presented in part 5.1 and tabulated and diagrammed accordingly.

The search engine was evaluated for 20 users for 20 days in equal conditions and the results are as following: (see Figure 2 and 3)

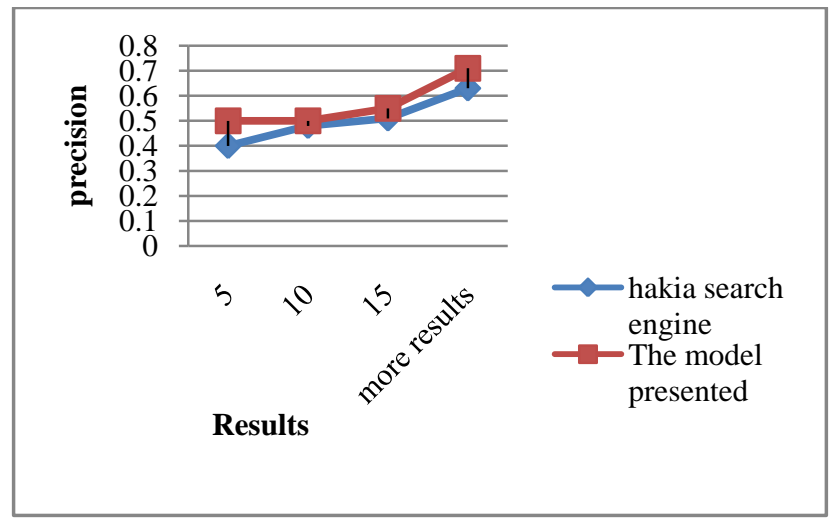

Fig 2: Average calculated precision for users search results

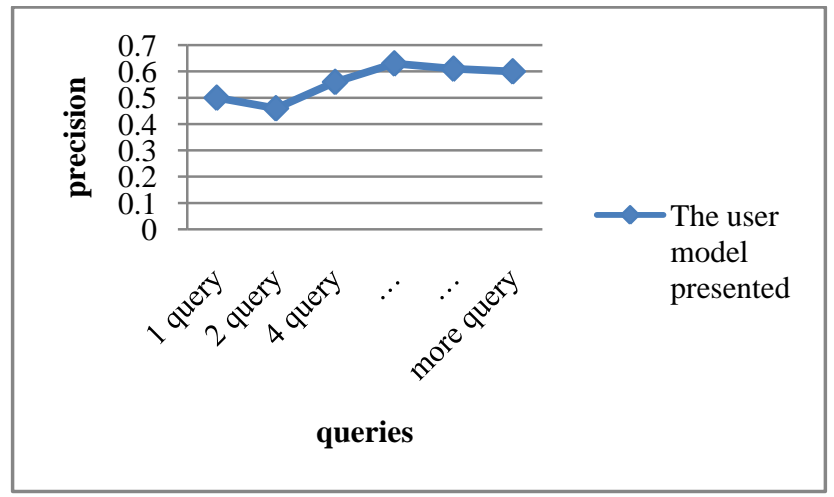

Fig 3: Average calculated precision for different queries by group of users

\section{CONCLUSION AND FUTURE WORKS}

Web is a huge dynamic environment with data being continuously added, updated and omitted. Therefore, it seems 
appropriate to filter the samples of information in order to develop web surfing systems. The conformation of incoming information with the user-interested model is a timeconsuming process because in the process of filtering the information, complicated displays of user's requirements are needed and the process of retrieving involves bigger span of time. This can answer the question why do sample information filtering not become extensively used tools for data retrieval from the web. Therefore, higher efficiency in filtering is equal to higher accuracy and smaller span of time that is tried to achieve in this paper.

In this paper, is developed a user model by examining different factors and research literature, analysing what contexts to be read and whart content to be contained. To foresee which pages are to be read on the basis of the previously read pages, is used a measurement scale of parallelism between new pages and old pages and the resemblance between the pages provided us with the criterion to estimate the user's interests. In this research, is suggested a technique to obtain and update user model by analysing the user's information from user's search history.

In this research, is concatenated on the embetterment of the efficiency of the results produced by the semantic search of updated user model. Since the low efficiency of personalized processes by the user model is due to low level factors and components by which user model is built up and the algorithm by which the model is updated. The suggested model in this paper can improve the efficiency of user models by investigating and modifying these cases; hence, the improvement of search results. Search results are enriched by the feedback from the user's search behaviors. The information implicitly elicited from the user cannot be directly used to describe the user's interests, but they are methodically interrelated. Some experiments were carried out to evaluate suggested technique and the results were indicative of user's satisfaction when they used their profiles for personalization.

The main question that must be considered in the future is which modeling technique, structure and application is most suitable regarding to the available data or how can choose depending on the situation, the best structure or technique for the application?

In the future, can be answered similar questions by the combinations of conditions (such as temporal or permanent information) in different researches. In addition to, user profiles can be progressed among the different applications in different domains.

\section{ACKNOWLEDGMENTS}

Our thanks to Turkey BİDEB program for supporting this paper.

\section{REFERENCES}

[1] Facca F.M., Ceri S., Armani J. and Demalde V. 2005. Building Reative Web Applications. Poster at WWW 2005, Chiba, Japan.

[2] Dominik Heckmann, Tim Schwartz, Boris Brandherm, Michael Schmitz and Margeritta von WilamowitzMoellendorff. 2005. Gumo - The General User Model Ontology. Lecture Notes in Computer Science, Volume 3538/2005, 428-432, DOI: 10.1007/11527886_58.

[3] Alessandro Micarelli, Fabio Gasparetti, Filippo Sciarrone, and Susan Gauch. 2007. Personalized Search on the World Wide Web. The Adaptive Web, pp. 195-230. Doi: 10.1007/978-3-540-72079-9_6 Key: citeulike: 1668950.
[4] Kappel G., Retschitzegger W., Kimmerstorfer E., Proll B, Schwinger W. and Hofer Th. 2002. Toward a Generic Customization Model for Ubiquitous Web Applications. Proceeding of the 2nd International Workshop on Web Oriented Software Technology (IWWOST), in conjuction with the 16th European conference on ObjectOriented Programming (ECOOP), Ma;aga, Spain, June 2002.

[5] Koch, N. 2001. Software Engineering for Adaptive Hypermedia Systems. Reference Model, Modeling Techniques and Development Process. PhD Thesis.

[6] Schwabe, D. and Rossi, G. 2001. A Conference Review System with OOHDM. In First Internacional Workshop on Web-Oriented Software Technology, 052001.

[7] Dicheva D., Aroyo, L. 2000. An approach to intelligent information handling in webbased learning environments. Proceedings of International Conference on Artificial Intelligence, CSREA Press, 1327-1333.

[8] Plaban kumar bhowmick, Sudeshna sarkar. 2010. Ontology based user modeling for personalized information access. Computer Science \& Engineering, Indian Institute of Technology, Kharagpur West Bengal, India-721302, ANUPAM BASU, International Journal of Computer Science and Application,Vol. 7 No. 1, pp. 1 22.

[9] Middleton, S. E., Alani, H., Shadbolt, N. R., and Roure, D. C. D. 2002. Exploiting synergy between ontologies and recommender systems. Proceedings of Semantic Web Workshop, At the Eleventh International World Wide Web Conference, 41-50.

[10] Peter Mika. 2008. Microsearch: An Interface for Semantic Search. CEUR Workshop Proceedings, ISSN 1613-0073, online at CEUR-WS.org/Vol-334, Ocata 1, 08003 Barcelona, Spain.

[11] Kalfoglou, Y., Domingue, J., Motta, E., Vargas-Vera, M., Shum, S. B. 1999. myPlanet: An ontology-driven webbased personalized news service. Proceedings of the IJCAI01 workshop on Ontologies and Information Sharing, 44-52.

[12] Gomez, J., Cachero, C., and Pastor, O. 2001. Conceptual Modeling of Device-Independent Web Applications. IEEE Multimedia Special Issue on Web Engineering, pp 26-39.

[13] Houben, G.J., Frasincar, F., Barna, P, and Vdovjak, R. 2004. Modeling User Input and Hypermedia Dynamics in Hera(Ed.). International Conference on Web Engineering (ICWE 2004), Lecture Notes in Computer Science, Vol. 3140, Springer-Verlag, Munich (2004) pp 60-73.

[14] Zhang, H., Song, Y., Song, H. 2007. Construction of Ontology-Based User Model for Web Personalization. Proceedings of the 11th international conference on User Modeling. 67-76.

[15] Zeng, Q., Zhao, Z., Liang, Y. 2009. Course ontologybased user's knowledge requirement acquisition from behaviors within e-learning systems. Computers \& Education, Elsevier Science Ltd. 53. 809-818.

[16] R.Guha, Rob McCool, Eric Miller. 2003. Semantic Search. Www 2003, May 20-24, Budapest, Hungary, ACM 1-58113-680. 
[17] Silvia Quarteroni, Suresh Manandhar. 2007. User Modelling for Personalized Question Answering. The University of York, York YO10 5DD, United Kingdom.

[18] My Yahoo! http://mysearch.yahoo.com.

[19] Google Personalized. http://labs.google.com/personalized.

[20] G. Jeh and J. Widom. 2003. Scaling personalized web search. In Proceedings of WWW 2003, pages 271-279.

[21] Feng Qiu, Junghoo Cho. 2006. Automatic Identification of User Interest for Personalized Search. University of California, Los Angeles, CA 90095, CA 90095.

[22] E. Volokh. 2000. Personalization and privacy. Communications of the ACM, 43(8):84-88.

[23] Liren Chen, Katia Sycara. 1998. WebMate: A Personal Agent for Browsing and Searching. Proceedings of the second international conference on Autonomous Agents.

[24] Keiichiro Hoashi, Kazunori Matsumoto, Naomi Inoue, Kazuo Hashimoto. 2000. Document Filtering Method Using Non-Relevant Information Profile, SIGIR.

[25] Dwi H. Widyantoro, Thomas R. Ioerger, John Yen. 1999. An Adaptive Algorithm for Learning Changes in User Interests. Eighth International Conference on Information and Knowledge Management (CIKM'99).

[26] Alexander Pretschner, Susan Gauch. 1999. Ontology Based Personalized Search. Proc. 11th IEEE Intl. Conf. On Tools with Artificial Intelligence, pp. 391-398, Chicago.

[27] Daniel Billsus, Michael J. Pazzani. 1999. A Personal News Agent that Talks, Learns and Explains. Third International Conference on Autonomous Agents.

[28] Raghavan, V.V., Sever, H. 1995. On the reuse of past optimal queries. In: Research and Development in Information Retrieval. 344-350. psu.edu/raghavan95reuse.html.

[29] T. Haveliwala., Topic-sensitive pagerank. 2002. In Proceedings of the Eleventh Int'l World Wide Web Conf.

[30] Peter W.Foltz. 1990. Using Latent Semantic Indexing for information filtering. COCS ' 90 Proceedings of the ACM SIGOIS and IEEE CS TC-OA conference on Office information systems, ACM SIGOIS Bulletin, Apr 1990.
[31] Pablo Castells1, et al. 2005. Self-tuning Personalized Information Retrieval in an Ontology-Based Framework. OTM Workshops 2005, p. 977-986.

[32] Xuehua Shen, Bin Tan, ChengXiang Zhai. 2005. Implicit User Modeling for Personalized Search. Proceeding of the 14th ACM international conference on Information and knowledge management, New York, ISBN: 159593-140-6.

[33] Chien Chin Chen, Meng Chang Chen, Yeali Sun. 2001. AWeb Document Personalization User Model and System. Proceedings of the seventh ACM SIGKDD international conference on Knowledge discovery and data mining, ISBN: 1-58113-391-X.

[34] Shian-Hua Lin, Chi-Sheng Shih, Meng Chang Chen, JanMing Ho, Ming-Tat Ko, and Yueh-Ming Huang. 2000 ACIRD: Intelligent Internet Documents Organization and Retrieval. Technical Report, IIS, Academia Sinica. To appear on IEEE Transactions on Knowledge and Data Engineering.

[35] Keiichiro Hoashi, Kazunori Matsumoto, Naomi Inoue, Kazuo Hashimoto. 2000. Document Filtering Method Using Non-Relevant Information Profile. SIGIR.

[36] Dwi H. Widyantoro, Thomas R. Ioerger, John Yen. 1999. An Adaptive Algorithm for Learning Changes in User Interests. Eighth International Conference on Information and Knowledge Management (CIKM'99).

[37] Shian-Hua Lin, Chi-Sheng Shih, Meng Chang Chen, JanMing Ho, Ming-Tat Ko, and Yueh-Ming Huang. 1998. Extracting Classification Knowledge of Internet Documents with Mining Term Associations: A semantic Approach. Proceedings of the 21st annual international ACM SIGIR conference on Research and development in information retrieval.

[38] Garrigós, I., Gómez, J., Barna,P., Houben, G.J. 2005. A Reusable Personalization Model in Web Application Design. Proceedings of The ICWE 2005 Workshop on Web Information Systems Modelling (WISM2005), Workshop at ICWE2005, International Conference on Web Engineering, pp. 40-49, Publ. University of Wollongong, School of IT and Computer Science, Sydney, Australia. 